\title{
Behavioral Modeling of Outphasing Amplification Systems
}

\author{
Keith Finnerty, Graduate Student Member, IEEE, John Dooley, Robin Wesson, \\ Mark P. van der Heijden, Member, IEEE, Mustafa Acar, Member, IEEE, \\ and Ronan Farrell, Member, IEEE
}

\begin{abstract}
This paper presents a comparison of existing and novel behavioral models targeted at the outphasing power amplifier (PA) architecture. A comprehensive comparison of ten modeling strategies is presented in the results. Novel techniques for outphasing PAs, such as vector switched and dual path time series, are also presented for the first time. Investigation of such techniques was driven by the analysis of outphasing operation at minimum output powers, demonstrating the generation of frequency-dependent amplitude and phase deviations, which can be difficult to characterize. The increased robustness was achieved at the cost of additional complexity; for practical implementation, time series coefficient reduction techniques were also evaluated. The results of all modeling approaches are experimentally validated for the wideband operation of an NXP 19-W GaN digital outphasing amplifier module. Considering computational complexity and accuracy for system-level modeling across all presented options, a subset of existing and new models is identified as best suited for modeling outphasing PAs.
\end{abstract}

Index Terms-Behavioral model, outphasing, radio frequency (RF) power amplifier (PA), Volterra.

\section{INTRODUCTION}

B EHAVIORAL models characterize complex nonlinear systems, providing a simplified computational means to include the nonideal effects in system-level simulations and linearization analysis. This approximation of radio frequency (RF) elements using appropriate algorithms can lead to a dramatic reduction in simulation time, in turn enabling the simulation of more complex transceiver systems; one such example is active antenna arrays. It is also of great benefit in system-level optimization, where large numbers of iterations are required, for example, in genetic algorithms or partial swarm optimization.

In most cases, RF power amplifiers (PAs) are nonlinear circuits. If not checked, the characteristic behavior of nonlinear PAs results in additional bit errors or unwanted spectral

Manuscript received May 7, 2015; revised July 24, 2015, January 25, 2016, July 3, 2016, and August 22, 2016; accepted August 28, 2016. Date of publication October 7, 2016; date of current version December 7, 2016 This work was supported by the Science Foundation Ireland under Grant 10/CE/I1853 and by HEA under PRTLI5 and is being co-funded by the Irish Government and the EU under Ireland's Structural Funds Programmes 2007-2013: Investing in Your Future.

K. Finnerty, J. Dooley, and R. Farrell are with the Department of Electronic Engineering, Callan Institute, National Institute of Ireland, Maynooth, Ireland (e-mail: kfinnerty@eeng.nuim.ie).

R. Wesson, M. P. van der Heijden, and M. Acar are with NXP Semiconductors N.V., 6534AE Nijmegen, The Netherlands.

Color versions of one or more of the figures in this paper are available online at http://ieeexplore.iee.org.

Digital Object Identifier 10.1109/TMTT.2016.2606618

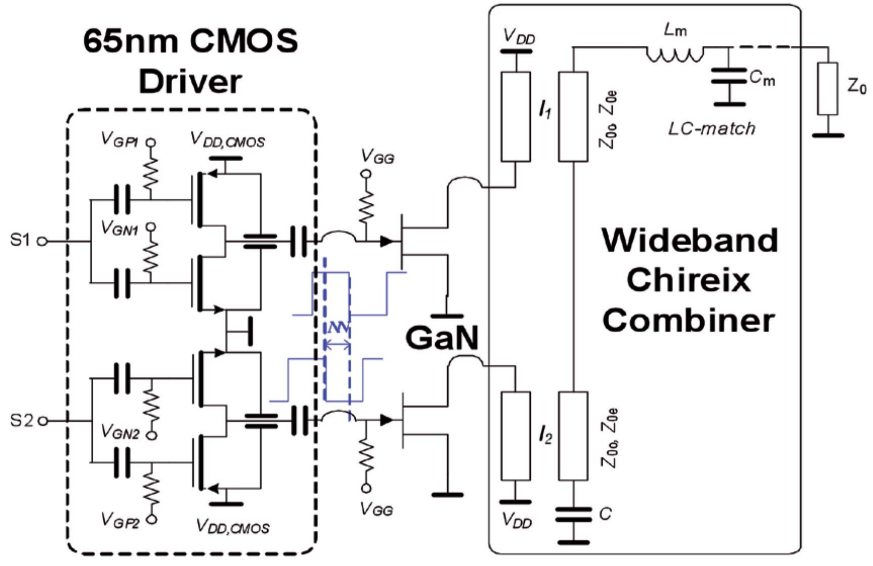

Fig. 1. Overview of digital outphasing amplifier with Chireix combiner used in this paper, originally presented in [6].

behavior. The PA typically consumes more power than any other single component within the base station transceiver. Therefore, it has the largest impact on transmitter efficiency. As such, amplifier topologies that can deliver increased efficiency are an important area of research. Modern efficient amplification systems, for example, envelope tracking [1], Doherty [2], and outphasing [3], have increased complexity in order to achieve more efficient operation compared with the classical PA architectures [4]. As a result of this increased complexity, circuit simulations of these newer PA architectures incur lengthy simulation times, especially when wideband modulated signals are applied as the input stimulus. Behavioral modeling of these systems can offer orders of magnitude reduction in simulation time. This in turn enables efficient system-level simulation, particularly when considering the distributed PA designs [5].

The concept of the outphasing amplifier is presented in [3] and a digital implementation of the outphasing system is given in Fig. 1. In this structure, an amplitude and phase modulated signal $S(t)$ is separated into two constant amplitude signals. Equations (1)-(4) denote the conversion of the amplitude to a phase shift inversely applied to the signal on each path

$$
\begin{aligned}
S(t) & =A(t) e^{(j \Phi(t))} \\
\varphi(t) & =\operatorname{arcos}\left(A(t) /\left(2 A_{\max }\right)\right) \\
S_{1}(t) & =A_{\max } e^{(j \Phi(t))} e^{(j \varphi(t))} \\
S_{2}(t) & =-A_{\max } e^{(j \Phi(t))} e^{(-j \varphi(t))}
\end{aligned}
$$


where $A_{\max }$ is half the maximum input amplitude, $\varphi(t)$ is the amplitude related phase shift, and $\Phi(t)$ is the phase angle of the input signal. The Chireix combiner topology offers the benefits of increased efficiency by operating both amplifiers in saturation. Operating the amplifiers in deep saturation is achieved with constant envelope modulated signals (3), (4). Constant envelope operation in this way improves the efficiency of the amplifier and negates the amplitude nonlinearity introduced by both the driver and final amplification stages.

The modeling challenge for outphasing PAs is more complex than classical PA structures. There are an increased number of mechanisms that can negatively impact on the output signal linearity. First, there are two distinct signal conversion processes that occur, namely, signal component separation and recombination. As highlighted in (3) and (4), there are also two separate signal paths within the amplifier structure each carrying unique signals. Gain, phase, and delay mismatch can arise between the two separate signal paths in the PA due to signal component separation or the difference in the two physical signal paths. Similarly, the frequency dependence of the load match between the two amplifiers and the combiner can give rise to dynamic signal imbalance. While models exist that can accurately describe static offsets, such as gain and phase delay, at the center operating frequency, newer models that encompass dynamic effects are only now being explored.

In this paper, we use a digital outphasing amplifier developed at NXP semiconductors [6] and shown in Fig. 9, the performance of which has been previously experimentally demonstrated for both the static and dynamic operations. The PA utilizes GaN transistors to implement efficient class-E switching amplifiers. The module also includes a CMOS driver stage; the inputs to the driver stage $S_{1}$ and $S_{2}$ are analog RF signals. The input driver stage hard limits the signal converting the analog signal to a pulsewidth (digital) representation of the signals phase. As discussed in [6], the output-matching network of the class-E amplifier is integrated into the Chireix combiner reducing the number of reactive components, in turn increasing output efficiency. The compact transformer-based combiner has increased operational bandwidth compared with a classical quarter-wave transmission line implementation. In this paper, the nonlinear effects associated with the wideband operation are examined as a quad carrier WCDMA signal is amplified from the outphasing system.

The resulting spectrum is shown in Fig. 2. In Fig. 3, the input-output amplitude characteristics (AM-AM) and the input-output phase characteristics relative to input amplitude (AM-PM) are plotted. Presenting the characteristics in this way highlights the nonlinear regions of a system. In Fig. 2, the visible spectrum contains significant out-ofband noise. The out-of-band noise is composed of nonlinearity amplitude effects and both the linear and nonlinear memory effects. Memory effects occur during the dynamic operation of the amplifier. In classic amplifier topologies, this has been attributed to parasitic within the device and biasing networks. However, in the outphasing topology, memory effects also result from path mismatch. In the Chireix combiner, these effects are relatively larger at lower output powers.

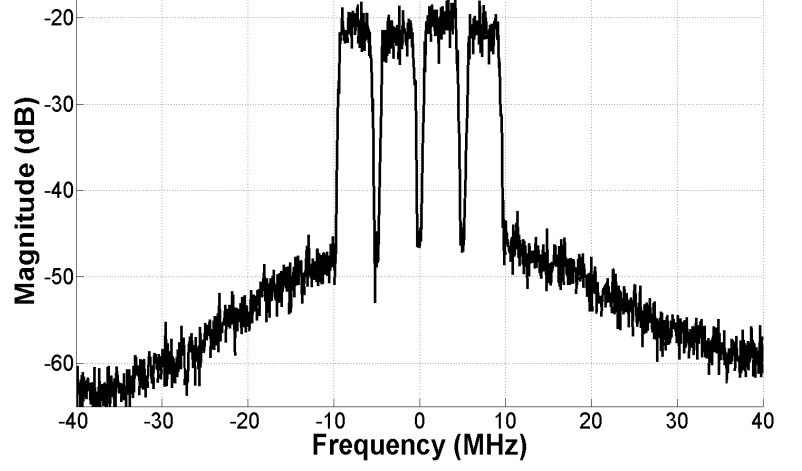

Fig. 2. Measured spectrum of uncorrected outphasing amplifier with quad carrier WCDMA signal, the figure demonstrates the relative noise level to the output signal power of the PA.

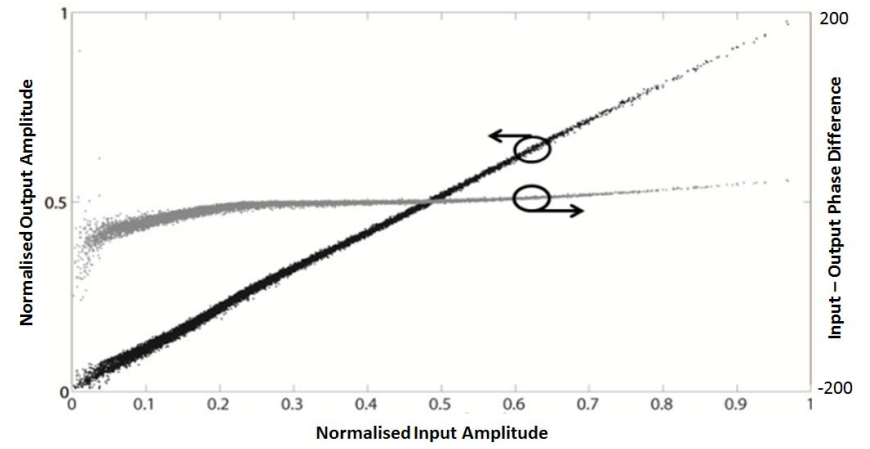

Fig. 3. Visualizing the relationship of the input amplitude versus output amplitude (AM-AM) and the input phase versus output phase as input amplitude changes (AM-PM).

In [7], a vector switch Volterra approach is used to linearize the NXP digital outphasing PA. This paper will extend on the work in [7], with more in-depth analysis of each model and enable a comparison with algorithms, which do not lend themselves to amplifier linearization. The models are evaluated with respect to characterization performance and computational complexity with the aim of identifying the optimum behavioral model for full system-level simulation. To this end, ten behavioral models are evaluated, including the first instance of a full dual path Volterra model applied to an amplifier structure as a behavioral model. Further to this, the Lasso algorithm is applied for the first time to reduce the number of model coefficients needed in outphasing PA models.

The remainder of this paper is as follows. The source of the outphasing amplifier memory at lower power operation due to dynamic signal imbalance is discussed in Section II. The current state-of-the art models for the outphasing architecture are introduced in Section III, along with a novel multi-input single-output (MISO) Volterra model for outphasing amplifier characterization. Section IV introduces the Lasso algorithm as a method of coefficient reduction. Section V will outline the measurement process, model extraction, and modeling results.

\section{Memory in An Outphasing Amplifier}

The outphasing PA requires balanced and calibrated signals in order to achieve linear recombination. Several analytical studies have been carried out [8], [9] demonstrating the impact 


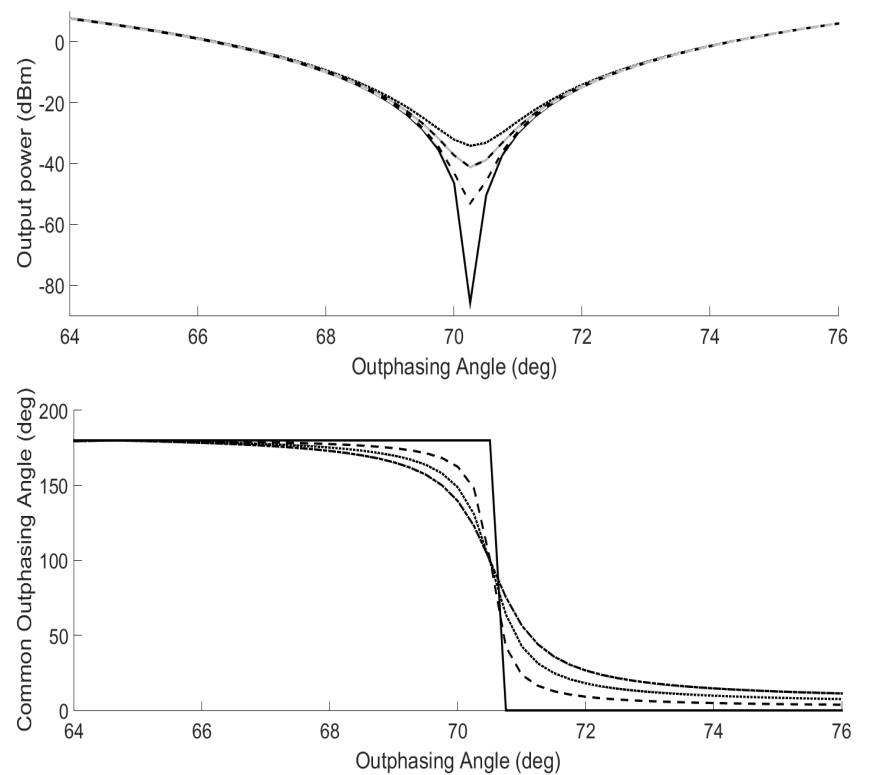

Fig. 4. Outphasing PA with Chireix combiner simulated in Agilent ADS. Analysis of dynamic range and signal phase relative to outphasing angle across frequency bandwidth of $100 \mathrm{MHz}$. The individual frequency steps are 12.5 MHz, opposing frequencies from the center frequency overlap. The center frequency of the simulations is $2.14 \mathrm{GHz}$.

of imbalances in outphasing amplifiers on linear operation. The main impact of an imbalance between the two signal paths is to limit the minimum achievable output power this in turn results in the loss of dynamic range. Fritzin et al. [10] directly relate the loss of dynamic range at the center frequency to an increase in nonlinearity, namely, an increase in ACPR. The assumption so far is that the dynamic range of the system at the center frequency is applicable to the outphasing system across the frequency of operation. This is only the case if the operational bandwidth of the system is relatively narrowband. The Chireix combiner in its traditional configuration is a tuned circuit [3]. Gerhard and Knoechel [11] perform a series of measurements on an outphasing amplifier employing a Chireix combiner over the predicted operation bandwidth. They present measurements demonstrating the variation of both outphasing angle and common signal phase with frequency. These results are replicated with a simulation of an outphasing PA and shown in Fig. 4. As outphasing angle is directly related to signal amplitude, the result is a deviation of both amplitude (AM-AM) and phase (AM-PM) with frequency at low output power. This is in line with the measurements of the digital outphasing PA shown in Fig. 3. Such a deviation is commonly referred to as memory in the analysis of a PA system.

As shown in Fig. 4, the effects are greatest at the lowest output powers where perfect vector cancelation is required. As the outphasing angle returns to higher output power regions, the output returns closer to the ideal output. Simulation is performed with a classical transmission line Chireix combiner, using ideal load compensation components. While the digital amplifier with the transformer-based combiner structure has a wider operational bandwidth, the same effects are still present; Fig. 5 shows the results for a sweep of outphasing angle

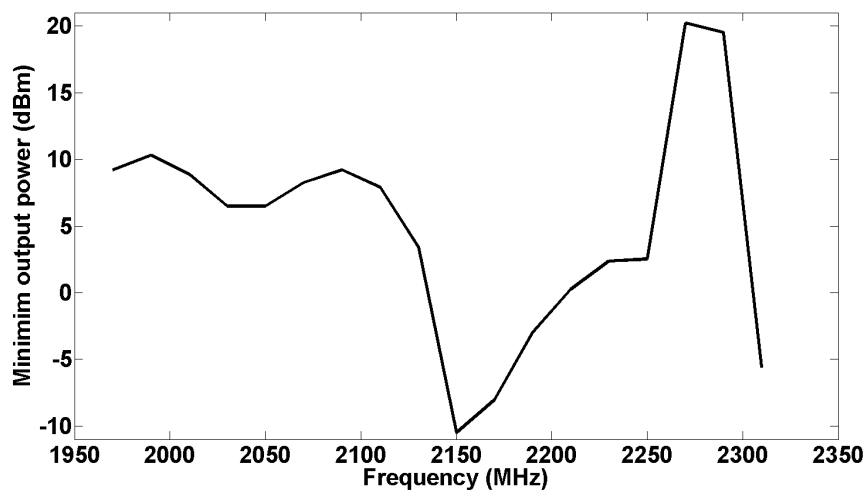

Fig. 5. Example of digital PA's minimum achievable amplitude measured across frequency, $\pm 180 \mathrm{MHz}$.

across a frequency of $\pm 180 \mathrm{MHz}$. Contrary to the extraction of behavioral models for the classical PA architectures, a behavioral model capable of characterizing the memory effects experienced at low power is required for an outphasing PA.

\section{BEHAVIORAL MODELS}

Behavioral modeling is the characterization of a system using a mathematical description, which is more compact and less computationally expensive than a full system simulation using equivalent circuit models. The outphasing PA is a complex system to model using traditional circuit simulators and therefore can greatly benefit from the computational reduction offered by behavioral modeling. As outlined in Sections I and II, the amplifiers operation and requirements for linearity deviate from that of traditional PA operation. Therefore, to generate an accurate outphasing amplifier model, these deviations must be taken into account. Determining the operation of the outphasing amplifier has been examined in great detail for static operation. Birafane and Kouki [9] extract both the amplitude and phase distortions generated by a Chireixbased outphasing PA. In this paper, the sources of nonlinearity are investigated, highlighting in particular the difficulties for low-power operation.

The signal component separator is assumed to be ideal for all the models evaluated in this paper. Nonlinearity between the input signals and the amplifier output is assumed to be inherent in the DUT. This assumption is based upon the small signal system being fully calibrated and the amplifier being statically characterized at the center frequency of operation; the resulting sweep returns a unique function for SCS decomposition. The amplifier can, therefore, be operated and subsequently modeled as an ideal outphasing system.

This paper will analyze a number of current Volterra-based amplifier models, namely, standard Volterra series, piecewise Volterra series, and vector switched Volterra series. This paper presents two novel amplifier models for the outphasing architecture, namely, vector switched Volterra using self-organizing maps (SOMs) and MISO Volterra series. In addition, the effectiveness of the Lasso method for coefficient reduction leading to increased model efficiency is presented. 


\section{A. Existing Behavioral Models}

In [11], a proposed method uses a lookup table (LUT) to modify the phase of the outphasing signals, to correct the amplifier. The LUT takes signal phase and signal frequency to find the correction factor for the output. The algorithm is a function of both input amplitude and frequency. In [12], a polynomial that maps input amplitude to phase correction for each outphasing path is derived. This is expanded upon in what is referred to as a direct outphasing model [13]; further, parameters that include amplitude and time delay are also introduced into the model. A two-path model incorporating memory taps is presented in [14]. Landin et al. [14] demonstrate the effectiveness of a memory capable model for outphasing PA. These existing behavioral models will be used to compare the proposed models in this paper.

1) Direct Outphasing Model: The direct outphasing model in (5) has been presented previously in [13] as an accurate and efficient model for an outphasing system. This system directly models the outphasing system where $\eta_{i}$ denotes the coefficients of the gain function, $\lambda_{i}$ denotes the coefficients of the phase function, and $\Delta_{i}$ is a delay factor that is applied to each path. The parameters $\eta_{i}$ and $\Delta_{i}$ are used to remove path mismatch from the system, $\lambda_{i}$ is the coefficients to a polynomial that alters phase in each outphasing path independently, correcting phase mismatch and linearizing the output signal simultaneously. Replicating the model in [13], the coefficients were initialized using the coefficient extraction process as outlined and optimized using a genetic algorithm [15], processed for 500 generations until optimization gradient approached zero and sufficiently low mean squared error was achieved

$$
\begin{aligned}
y(n)= & g_{1}\left(s\left(t_{n}-\Delta_{1}\right) ; \eta_{1}\right) e^{j p_{1}\left(s\left(t_{n}-\Delta_{1}\right) ; \lambda_{1}\right)} s_{1}\left(t_{n}-\Delta_{1}\right) \\
& +g_{2}\left(s\left(t_{n}-\Delta_{2}\right) ; \eta_{2}\right) e^{j p_{2}\left(s\left(t_{n}-\Delta_{2}\right) ; \lambda_{2}\right)} s_{2}\left(t_{n}-\Delta_{2}\right) .
\end{aligned}
$$

2) Amplitude Distortion Outphasing Model: Landin et al. [14] present an outphasing amplifier model capable of characterizing frequency-dependent amplifier effects. They describe the model as AM distortion model based upon parallel Hammerstein function. The model is presented in (6) and (7). In this model, a function uses the outphased signals $s_{i}(n)$ and the input signal magnitude $\|s(n)\|$ to the outphasing paths. The model uses a parallel linear and nonlinear function to accurately describe the frequency effects of both the amplifiers and the output combiner

$$
\begin{aligned}
y(n)= & g_{1}\left(s(n) ; \tau_{1}\right) s_{1}(n)+g_{2}\left(s(n) ; \tau_{2}\right) s_{2}(n) \\
y_{i}\left(s(n) ; \gamma_{i}\right)= & \sum_{m=0}^{M_{\text {lin }}} \gamma_{i, 0, m} s_{i}(n-m)+\sum_{p=1}^{P} \sum_{m=0}^{M_{\text {nonlin }}} \\
& \times \gamma_{i, 0, m} s_{i}(n-m)\|s(n-m)\|^{p} .
\end{aligned}
$$

\section{B. Classical Volterra Series Model}

The Volterra series is a robust algorithm capable of estimating nonlinear memory effects and has been previously applied to model and linearize power amplification systems [16]. To model the outphasing amplifier, the input is the signal to the outphasing decomposition function, and this is a limiting factor in the models ability to correct individual path mismatch. The equation for the Volterra series is presented in (8), where $\tilde{x}(n)$ and $\tilde{y}(n)$ are the input and output complex signal samples and $\tilde{h}_{2 p-1}\left(q_{1}, \ldots, q_{2 p-1}\right)$ denotes the discrete time Volterra kernels. $P_{0}=2 P-1$ is the kernel order, $M$ is the memory depth, and ${ }^{*}$ denotes the conjugate transpose

$$
\begin{aligned}
\tilde{y}(t)= & \sum_{p=1}^{P_{0}} \sum_{q_{1}=0}^{M} \sum_{q_{2}=q_{1}}^{M} \ldots \sum_{q_{p}=q_{p-1}}^{M} \sum_{q_{p+1}=0}^{M} \sum_{q_{p+2}=q_{p+1}}^{M} \ldots \sum_{q_{2}-1=q_{2}-2}^{M} \\
& \times \tilde{h}_{2 p-1}\left(q_{1}, q_{2}, \ldots, q_{2 p-1}\right) \prod_{j_{1}=1}^{p} \tilde{x}\left(t-q_{j_{1}}\right) \prod_{j_{2}=p+1}^{2 p-1} \tilde{x} \\
& *\left(t-q_{j 2}\right) .
\end{aligned}
$$

\section{Multiple-Input Single-Output Volterra Series}

In [17], a method of identifying multi-input nonlinear systems is presented. Using a complex nonlinear time series expanded for an MISO system, a more comprehensive model of the outphasing amplifier can be derived in comparison to standard Volterra series. The MISO model was initially developed in [17], and chosen for this application as the structure can be arranged to resemble the physical layout of the outphasing PA. As shown in Fig. 4 and (7) and (8), a two path structure is mathematically similar to the outphasing amplification system, each Volterra series, $h l$ and $h 2$, is capable of modeling a single-input nonlinear system, as outlined in Section III-A. The cross kernel is capable of modeling the interaction between the two amplification paths and the memory function of the power combiner. The capabilities of a nonlinear time series have been extended for multiinput systems by including cross terms that can describe the interaction between the individual signal paths. As described in the outphasing amplifier theory [3] and (1)-(4), each amplifier interacts with the load. Given that there is finite isolation between amplification paths and the reflection, which occurs between the amplifiers and the load [11], each amplifier also acts on one another. This interaction as well as crosstalk distortion can be modeled using cross terms in a time series MISO model

$$
\begin{aligned}
\tilde{y}(n)= & \sum_{p=1}^{P_{0}} \bar{h}_{p}[\tilde{x}(n)] \\
\tilde{y}(n)= & \sum_{p=1}^{P_{0}} \bar{h}_{p 1}\left[\tilde{S}_{1}(n)\right]+\sum_{p=1}^{P_{0}} \bar{h}_{p 2}\left[\tilde{S}_{2}(n)\right] \\
& +\sum_{p=1}^{P_{0}-1} \bar{h}_{p 12}\left[\tilde{S}_{1}(n) \tilde{S}_{2}(n)\right] .
\end{aligned}
$$

Expanding a single-input single-output model for a two-path implementation requires a time series in both paths and an additional set of kernel coefficients for cross products between each path. Equation (8) shows the kernel composition of the two-path model, while it contains significantly more weights than the single path Volterra series; the additional complexity provides a more accurate model. As stated in [17], the number of inputs does not limit the 
structure to a two input model; however, dimensionality will dramatically increase with the number of inputs.

$S_{1}$ is the input from path one of the outphasing amplifier, and $S_{2}$ is the input from path two. The polynomial extraction of the input signal associated with Volterra series expansion is indicated for each path as $\tilde{S}_{1}$ and $\tilde{S}_{2}, \bar{h}_{p}$ are the linear weights applied to the series expansion signals $\tilde{S}_{1}$ and $\tilde{S}_{2}$. " $\sim$ " indicates a vector of nonlinear, polynomial/Volterra inputs, and " $h$ " indicates the vector of linear weights. The cross terms of the model $h_{p 12}$ consist of a square matrix. In line with the model in [17], the matrix $h_{p 12}$ nonlinear components is one order lower than the standard time series $h_{1}$ and $h_{2}$.

\section{Segmented Volterra}

An objective of this paper is to highlight the effects that occur at lower output power levels for outphasing amplifiers. At higher power, the spreading effect associated with memory is less pronounced and a more traditional polynomial nonlinearity is dominant. It is this variation in characteristic performance within the amplifier that has led to the investigation of segmented modeling methods for single-input, single-output modeling.

Nonlinear models using piecewise segmentation have been successfully used in conjunction with both polynomial [18] and time-series [19] functions for nonlinear modeling for PAs. Afsardoost et al. [20] present an improvement to the previous segmentation techniques. This process generates a 2-D feature space where clustering is also called vector quantization. The individual segments are analyzed and individual functions can be extracted. As such, each segment can be characterized using a reduced nonlinear order and memory depth compared with characterizing all segments using a single function. A Volterra series can be applied to each of the individual segments. A 2-D feature space is generated using $\{\operatorname{mag}(x(n)), \operatorname{mag}(x(n-m))\}$, where $x$ is the input signal, $n$ is the current time step, and $m$ is a positive integer that generates a constant delay factor. Afsardoost et al. [20] apply $k$-means clustering algorithm to perform automated vector quantization.

1) Segmented Volterra Using k-Means: $K$-means is a commonly used technique for both clustering and segmentation. The algorithm is applied to a multidimensional feature space and partitions it into $k$ clusters defined with hard boundaries. At the center of each cluster is a point called a centroid. To initialize the algorithm, the centroids are evenly distributed over the feature space. The centroids are iteratively repositioned to find the center of the clusters in the feature space. The algorithm iteratively updates the centroids position using a distance metric between the centroid and the points in its region. The algorithm continues until a predetermined threshold of movement has been reached. The results are saved in a codebook or LUT, which is later used as the quantization function.

2) Segmented Volterra Using Self-Organizing Maps: SOMs have been identified as a more robust clustering algorithm when compared with $K$-means [21]. A special form of neural networks, such as the SOM, can be implemented using a numerical function or with cluster LUT method similar to

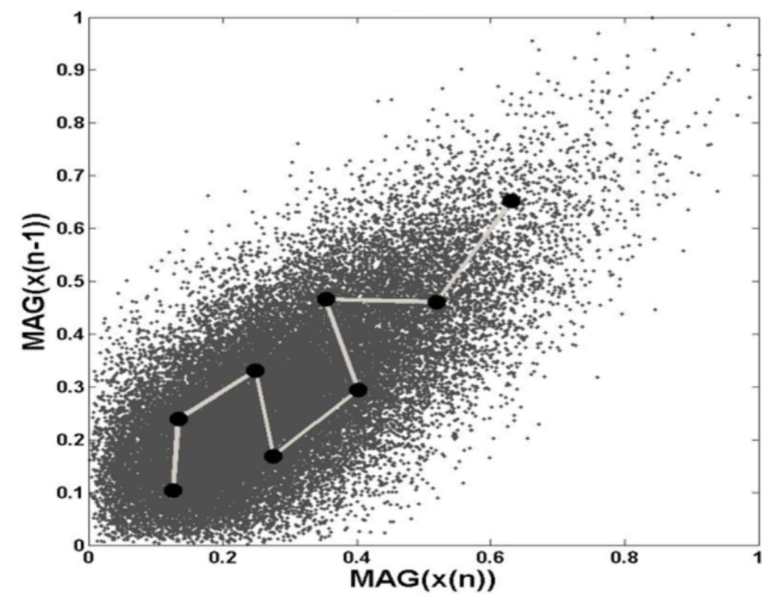

Fig. 6. SOM weights are highlighted in black, the input data feature space is highlighted in gray. An example of SOM vector quantization for the input data $x(n)$. The weight locations are the centroids of the vector quantization process.

the $k$-means algorithm. The structure of the SOM is outlined in [22]. The vector switched approach has shown that the segmentation of the time series algorithm can be beneficial, improving the capability of behavioral models. In this paper, the objective is to demonstrate that additional performance can be gained through the optimization of the vector quantization algorithm. An example for the input amplitude feature space and the results from the clustering algorithm are shown in Fig. 6.

The Kohonen algorithm, an unsupervised learning process, is used to train the SOM as a vector quantization algorithm. The optimization of the SOM is achieved by determining the fewest number of neurons capable of accurately separating the feature space. A drawback to SOM is an increase in computational complexity of the training algorithm. In [23, eqs. (10) and (11)], a method to compare both $K$-means and SOM directly is provided

$$
\begin{aligned}
\mathrm{SOM} & =O\left(\left(N^{2} * n_{O}\right) * 2+\left(N * n_{O} * \log \left(n_{O}\right)\right)\right) \\
K_{\mathrm{MEANS}} & =O\left(n_{O}\right) .
\end{aligned}
$$

In (11), $N$ is the dimension of the feature space and $n_{O}$ is the number of clusters in the feature space. For a comparison of computational complexity eight centroids and it is a 2-D feature space. The SOM has an increase in the computational complexity of 12 times that for the $K$-means algorithm. This is an increase per training iteration; however, the robustness of the SOM means that the algorithm requires fewer iterations to reach centroid stability. In this example, SOM required $25 \%$ fewer iterations compared with the $K$-means algorithm. The net result is that the SOM is still more computationally intensive; however, as the results in Section IV will show, this leads to an increase in accuracy.

\section{Time Series Coefficient Reduction}

Coefficient reduction tools enable the implementation of efficient models, which can be easily interoperated without sacrificing accuracy. With a large number of coefficients, a system can be accurately modeled; however, overfitting 


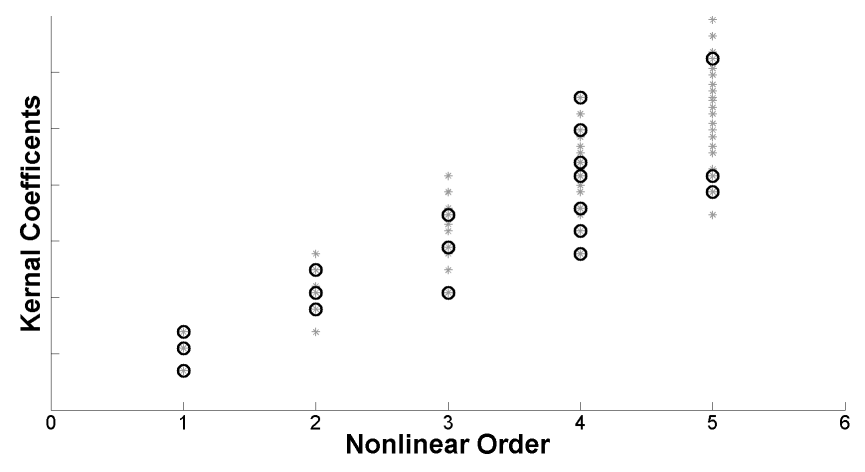

Fig. 7. Visualization of the coefficient reduction with the Lasso function. The gray points represent the full Volterra kernel. The black circles highlight the chosen coefficients for the reduced model.

may be of concern. By ranking the model parameters in the order of importance and selecting a sufficient number to accurately model the system, the potential of overfitting can be reduced. Applied to behavioral modeling, coefficient reduction has a direct impact on computational complexity, reducing the computational cost of the implemented model. This is a desirable trait when applied to large modeling structures. The Lasso is a robust high-performance method of parameter reduction; the algorithm achieves this by utilizing a combined method of regression and shrinkage. It has been shown that when implemented with Volterra series, significant reductions can be achieved [24] and for PA modeling, this can greatly reduce the computation complexity to implement the resulting model [25].

The Lasso time series definition is as follows: $\boldsymbol{x}^{t}=$ $\left(x_{t, 1}, \ldots, x_{t p}\right)^{T}$, where $\boldsymbol{x}^{t}$ are the kernels of the input and $y_{t}$ is the target output of the function. Some assumptions must be made: the function targets $\left(y_{t}\right)$ are independent and $x_{t p}$ is normalized so that the mean of $x_{t p}$ is 0 and the mean of $x_{t p}^{2}$ is 1 . These are common to regression functions. The Lasso estimate as defined in [26] is as follows:

$$
\begin{aligned}
&(\hat{\alpha}, \hat{\beta})= \arg \min \left\{\sum_{t=1}^{N}\left(y_{t}-\alpha-\sum_{p} \beta_{p} x_{t p}\right)^{2}\right\} \\
& \text { s.t. } \sum_{p}\left|\beta_{p}\right| \leq k
\end{aligned}
$$

where the tuning parameter, $k$ must be greater than zero, given that the mean of $y_{t}$ is also zero. During the optimization of parameters, the value of $k$ is used to tune the algorithm to find a compromise between model accuracy and number of coefficients. In this paper, the optimization of $k$ is performed as outlined in [26]. A vector of reasonable values is analyzed using the generalized cross validation technique [27]. The vector consists of $N$ values equally distributed from $k_{0}$ to 0 , where $k_{0}$ corresponds to the least squares solution with zero shrinkage, and $k_{0}=\sum\left|\beta_{p}^{0}\right|$, where $\beta_{p}^{0}$ is the least squares solution.

The optimum value of $k$ is dependent on the characteristics of the system being identified. PAs are largely deterministic and in communications systems, the input and output data are highly correlated. Given this application, the Lasso lends itself well to reducing the number of coefficients while maintaining

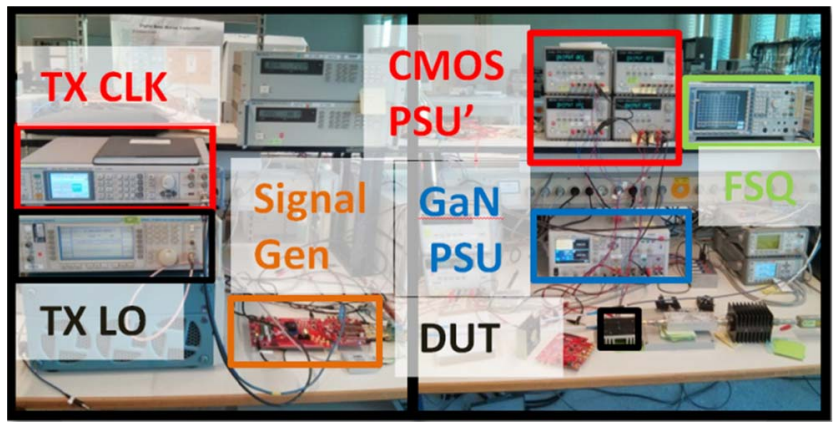

Fig. 8. Outphasing test-bench.

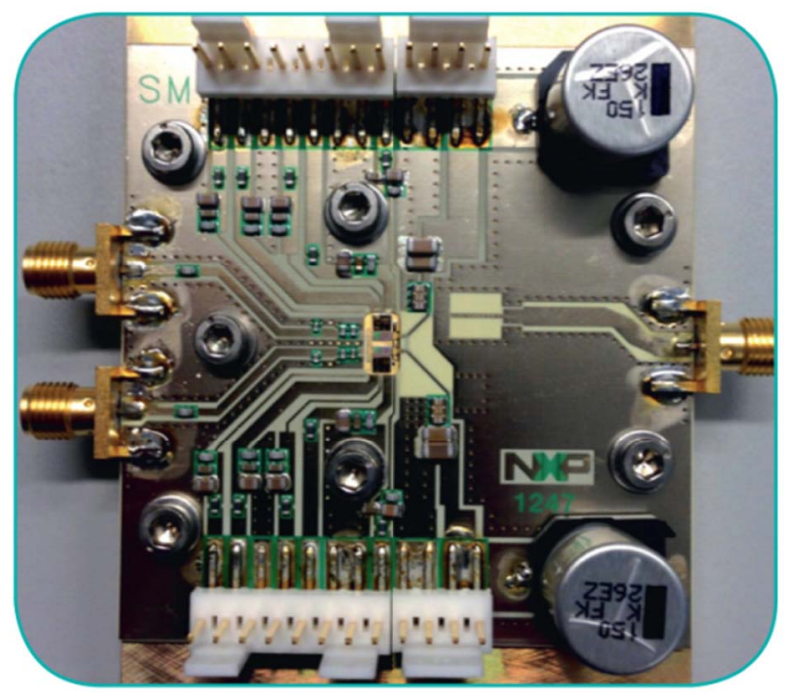

Fig. 9. DUT-digital outphasing PA.

accuracy. In Fig. 7, coefficient reduction is visualized for a full Volterra series.

\section{Measurements and Results}

The measurement setup uses a pattern generation board capable of driving a dual transmit chain DAC board; the DAC can output a $16 \mathrm{~b}$ signals of bandwidths up to 614.4 MSPS. The system is capable of generating dual wideband quadrature signals, supplying the amplifier with inputs $S_{1}$ and $S_{2}$. Signal capture is performed using Rohde and Schwarz FSQ vector signal analyzer. Signals are captured and analyzed over a bandwidth of $122.88 \mathrm{MHz}$. The system is calibrated to remove the following:

1) quadrature signal imbalance;

2) local oscillator feed through;

3) amplitude imbalance between outphasing paths;

4) amplitude and phase ripple over frequency.

The offsets are for the signal generation system alone, providing the amplifier with a frequency balanced and linear input to ensure that the measurements only included nonlinear effects from the DUT. The amplifier in Fig. 9 was fully operational for a period of time prior to measurement to ensure it was at steady state so that the drivers and the amplifier were at a stable temperature. The amplifier is characterized with a phase angle sweep to determine the maximum and 


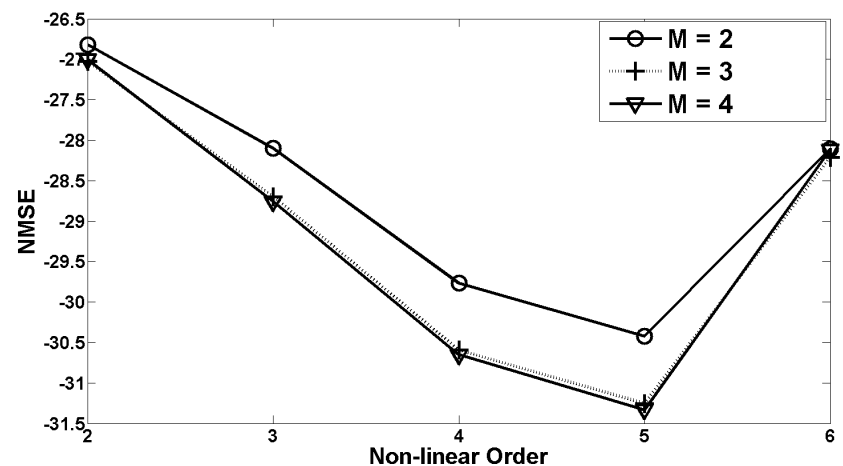

Fig. 10. Dual path Volterra series accuracy (NMSE) is compared over different model dimensions, each line represents the memory depth and each data point represents the maximum nonlinear order.

minimum outphasing angles. These are stored in an LUT and used to ensure that maximum dynamic range is achieved during dynamic operation. No other offsets are applied to the amplifier. To verify the generalization capabilities of the models, two data sets were used, namely, a training set and a test set. Both are quad carrier WCDMA signals, with 9-10 dB peak to average power ratio. The signals were independently generated using Agilent Advanced Design Systems.

Analysis was carried out in MATLAB (2013), utilizing a Core i 7 - 2600 CPU and 8 GB of memory. Signal conditioning and all preprocessing steps applied before the final coefficients are extracted. This includes piecewise and vector switched segmentation based on the input signal only and coefficient reduction based on the input and output signals. The time series is split into a 50\% train and 50\% test set with 16000 data points in each. The final set of weights is extracted using least squares estimation with QR decomposition [28]. The optimal model dimensions were determined by sweeping model order, number of memory terms, and number of segments. The range of nonlinear order was swept from third to ninth order, using only odd orders. The memory depth was swept from two to six using all terms and the vector quantization segments were swept from 5 to 20 using all terms. For each model, the maximum accuracy occurred as a knee point, after which no additional accuracy was observed. This is demonstrated with an example in Fig. 10. In the cases where higher model dimensions do not yield an appreciable performance increase, the most computationally efficient model is chosen for evaluation. Normalized mean-square error (NMSE) was used to determine the best performing model. NMSE is outlined in (14). NMSE is chosen as a figure of merit for the outphasing amplifier due to the fact that the normalized error weights the effects of memory and path mismatch at lower output powers, which is difficult to characterize with the nonlinearity at high output powers, which is relatively easy to characterize

$$
\begin{aligned}
\text { MSE } & =\frac{\sum_{n=1}^{N}\left|d_{k}(n)-y_{k}(n)\right|^{2}}{N} \\
\text { NMSE } & =\frac{\sum_{n=1}^{N} \frac{\left|d_{k}(n)-y_{k}(n)\right|^{2}}{\left|d_{k}\right|^{2}}}{N}
\end{aligned}
$$

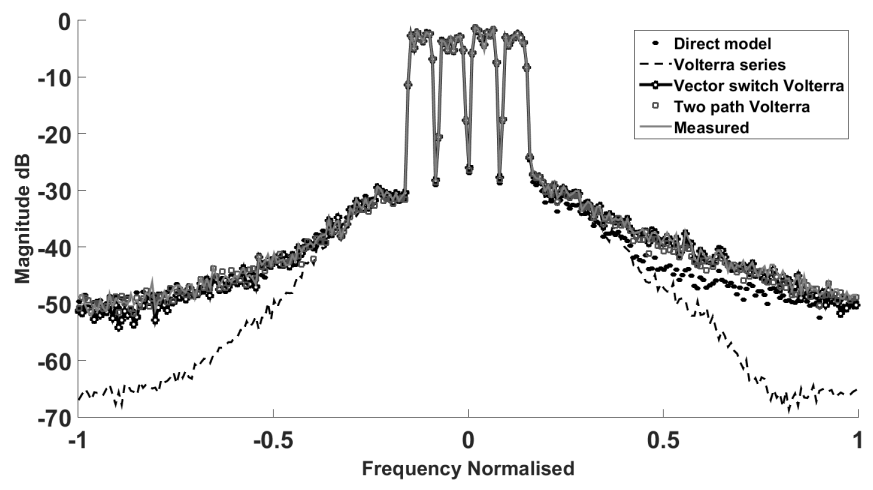

Fig. 11. Spectral plot of model test data.

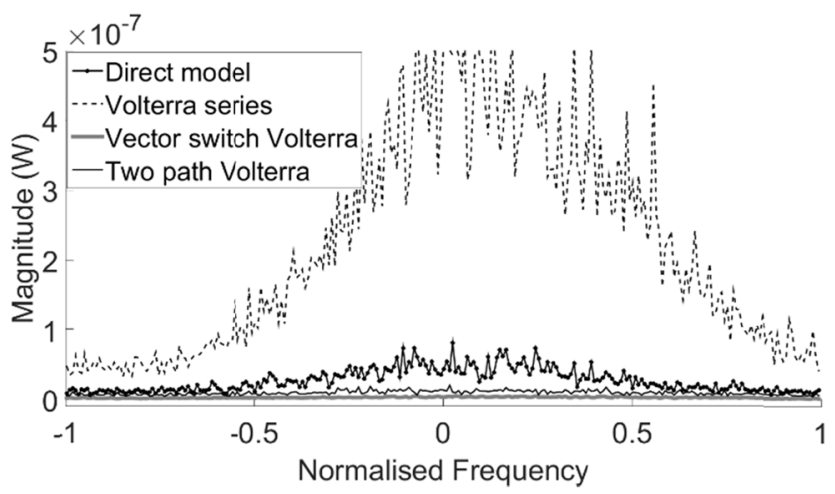

Fig. 12. Spectral analysis of modeling error squared. The error is presented in a linear scale.

$$
\operatorname{ACEPR}=\frac{\int_{\text {adj }}|E(f)|^{2} d f}{\int_{\text {ch }}|D(f)|^{2} d f}
$$

where $d_{k}$ is the measured signal and $y_{k}$ is the model output. The accuracy of the models spectral estimation is carried out using adjacent channel error power ratio (ACEPR) [29]. The ACEPR calculation is outlined in (16). $E(f)$ is the Fourier transformation of the model error and $D(f)$ is the Fourier transform of the output signal. The results are presented in Table I, which outlines training time for each model as well as the final number of coefficients.

The error for each model is evaluated with a test data set, and an independent data set from the training sequence. Figs. 11 and 12 show the spectral accuracy of the behavioral models. The results are, as expected, in line with the NMSE results in Table I.

\section{A. Volterra Series}

The limitations of the Volterra series model have been highlighted in the results in Table I and Fig. 11. The Volterra series is the basis for each of these models; however, its performance is fundamentally limited to narrowband characterization, as seen in Fig. 11. It cannot model out-of-band nonlinear beyond $\pm 20 \mathrm{MHz}$, at which point the majority of the distortion is associated with outphasing noise. Outphasing noise is a function that varies with output power as seen in Fig. 3 and highlighted in Section II. 
TABLE I

Results From Modeling ANALysis

\begin{tabular}{|l|r|r|r|r|r|r|r|r|}
\hline Model & $\begin{array}{r}\text { Weights } \\
\text { (total) }\end{array}$ & $\begin{array}{r}\text { Training } \\
\text { Time } \\
\text { seconds) }\end{array}$ & $\begin{array}{r}\text { Model } \\
\text { order }\end{array}$ & $\begin{array}{r}\text { Model } \\
\text { segments }\end{array}$ & $\begin{array}{r}\text { Memory } \\
\text { Taps }\end{array}$ & $\begin{array}{r}\text { Test } \\
\text { MSE }\end{array}$ & $\begin{array}{r}\text { Test } \\
\text { NMSE }\end{array}$ & ACEPR \\
\hline Volterra & 70 & 13.30 & 7 & NA & 3 & $-39 \mathrm{~dB}$ & $-26.7 \mathrm{~dB}$ & $-36.8 \mathrm{~dB}$ \\
\hline Volterra - Lasso & 38 & 47.70 & 7 & NA & 3 & $-39 \mathrm{~dB}$ & $-26.2 \mathrm{~dB}$ & $-37.0 \mathrm{~dB}$ \\
\hline Volterra Piecewise & 833 & 9.70 & 5 & 7 & 3 & $-47 \mathrm{~dB}$ & $-34.0 \mathrm{~dB}$ & $-46.8 \mathrm{~dB}$ \\
\hline VSV K-means & 1190 & 10.90 & 5 & 10 & 3 & $-48 \mathrm{~dB}$ & $-35.7 \mathrm{~dB}$ & $-49.0 \mathrm{~dB}$ \\
\hline VSV SOM & 1190 & 11.57 & 5 & 10 & 3 & $-49 \mathrm{~dB}$ & $-36.2 \mathrm{~dB}$ & $-49.2 \mathrm{~dB}$ \\
\hline VSV SOM - Lasso & 616 & 87.00 & 5 & 10 & 3 & $-45 \mathrm{~dB}$ & $-33.2 \mathrm{~dB}$ & $-46.0 \mathrm{~dB}$ \\
\hline Two Path Volterra & 256 & 23.90 & 5 & NA & 3 & $-44 \mathrm{~dB}$ & $-31.1 \mathrm{~dB}$ & $-43.1 \mathrm{~dB}$ \\
\hline Two Path Volterra - Lasso & 23 & 133.60 & 5 & NA & 3 & $-43 \mathrm{~dB}$ & $-30.68 \mathrm{~dB}$ & $-43.1 \mathrm{~dB}$ \\
\hline Direct mode - GA optimization & 16 & 492.00 & 6 & NA & NA & $-41 \mathrm{~dB}$ & $-28.5 \mathrm{~dB}$ & $-40.5 \mathrm{~dB}$ \\
\hline Amplitude distortion model & 50 & 5.00 & 6 & NA & {$\left[1 \mathrm{M}_{\text {lin }}\right.$,} & $-43 \mathrm{~dB}$ & $-31.5 \mathrm{~dB}$ & $-43.9 \mathrm{~dB}$ \\
\hline
\end{tabular}

\section{B. MISO Volterra Series}

The multi-input Volterra model is comparable to the direct outphasing model and the amplitude distortion model. These models use the outphasing signals as an input and therefore can model the nonlinear effects associated with a single path. In comparison with the existing models, this novel two-path Volterra model had additional cross terms as well as memory taps in each path that provide a more comprehensive model when compared with the direct model. This is carried out at the expense of increased computation in the forward path. The results from Table I demonstrate that the two-path model has increased accuracy when compared with the direct outphasing model. However, it is not as accurate as the amplitude distortion model. The dual input Volterra model and the amplitude distortion model have comparable performance when comparing spectral characteristics.

\section{Segmented Volterra Series}

There are three segmented time series models analyzed in this paper: piecewise Volterra, $K$-means, and SOM vector switched Volterra. Both vector switch models had an optimum number of ten segments, while piecewise had an optimum number of seven segments. Of the segmented models, VSV SOM has an NMSE performance of $-36.2 \mathrm{~dB}$, a clear advantage over the next best nonsegmented approach, two-path Volterra with $-31.1 \mathrm{~dB}$. These results remove any ambiguity from the model selection where the highest accuracy is required; a segmented approach appears to be the best. The individual Volterra series used fifth-order nonlinear components with three memory taps.

\section{Lasso}

The Lasso algorithm enabled an increase in computational efficiency in the Volterra, dual input Volterra, and vector switched Volterra models. The algorithm enabled a reduction of coefficients by $68 \%, 90 \%$, and $48 \%$, respectively. The remaining coefficients form a new amplifier model structure in which accuracy is generally retained and in some circumstances increasing the models generality. In Table I, this is demonstrated as Lasso models were able to achieve accuracy within $1 \mathrm{~dB}$ NMSE of the original model. The tradeoff for this reduction in coefficients is an increased model extraction time.

\section{CONCLUSION}

This paper has presented behavioral models for an outphasing amplifier system operating with a multicarrier modulated signal. Initial analysis outlines the nonlinear characteristic which, we believe, limits the accuracy of existing behavioral models. The models that are outlined in this paper target the errors at low power output without compromising accuracy at high output powers. Existing amplifier modeling techniques, Volterra series and vector switched Volterra series, were analyzed, and it was demonstrated that a single-input topology is capable of modeling an outphasing amplifier; however, a standard time series cannot fully characterize the system. Advancements on the vector switch approach demonstrate the benefit that can be achieved through the optimization of the vector quantization algorithm; the SOM algorithm has been applied as a more robust solution. The comparison also includes a novel two-path Volterra structure, which more closely resembles the physical layout of the outphasing PA. From Table I, it is evident that the multisegment Volterra provides the most accurate outphasing amplifier model. This is achieved by applying individual nonlinear memory time series to different amplifier operating regions based upon signal characteristics. Using signal amplitude as a method of segmentation enables the characterization of the frequency and amplitude-dependent nonlinear effects demonstrated in Section II.

The MISO Volterra model provides modeling accuracy, which matches or exceeds existing outphasing modeling strategies. However, the number of coefficients greatly increases with the dual input Volterra series. The Lasso algorithm reduced the MISO Volterra structure to 23 coefficients, a saving of $90 \%$ in computational load when implementing the model, while maintaining a performance advantage over the direct outphasing model, which requires 16 coefficients. 
Depending on the system being implemented a tradeoff of computation load and accuracy can be chosen, for single amplifier systems and models requiring the highest level of accuracy multisegment time series and in particular SOM vector switch Volterra is the best option. However, in larger transmitter system structures where computational complexity is a concern, such as active antenna arrays, a two-path solution provides the best compromise. The amplitude distortion model's accuracy of the time domain signal is better at the cost of additional coefficients. In the frequency domain, the MISO Volterra series including the Lasso algorithm provides comparable ACEPR performance to the amplitude distortion model.

This paper presents and evaluates alternative modeling strategies for the outphasing PA. As well as traditional two-path modeling approaches, the capability of single input-output behavioral models are examined. The aim of all the models is to accurately describe the effects of dynamic path mismatch. The models presented have the ability to achieve increased characterization performance, lending to more accurate behavioral-level system simulations.

\section{ACKNOWLEDGMENT}

The authors gratefully acknowledge the support of Science Foundation Ireland, HEA, the Irish Government, and the EU.

\section{REFERENCES}

[1] J. Staudinger et al., "High efficiency CDMA RF power amplifier using dynamic envelope tracking technique," in IEEE MTT-S Int. Microw. Symp. Dig., Boston, MA, USA, Jun. 2000, pp. 873-876.

[2] B. Kim, J. Kim, I. Kim, and J. Cha, "The Doherty power amplifier," IEEE Microw. Mag., vol. 7, no. 5, pp. 42-50, Oct. 2006.

[3] H. Chireix, "High power outphasing modulation," Proc. Inst. Radio Eng., vol. 23, no. 11, pp. 1370-1392, Nov. 1935.

[4] S. C. Cripps, RF Power Amplifiers for Wireless Communications, 2nd ed. Norwood, MA, USA: Artech House, 2006.

[5] B. L. Ng et al., "Fulfilling the promise of massive MIMO with 2D active antenna array," in Proc. Globecom Workshops, Dec. 2012, pp. 691-696.

[6] M. P. van der Heijden, M. Acar, J. S. Vromans, and D. A. Calvillo-Cortes, "A 19W high-efficiency wide-band CMOS-GaN class-E Chireix RF outphasing power amplifier," in IEEE MTT-S Int. Microw. Symp. Dig., Jun. 2011, pp. 1-4.

[7] K. Finnerty, R. Wesson, J. Dooley, M. Acar, M. P. van der Heijden, and R. Farrell, "Linearization of an outphasing amplifier for wide-band multi-carrier signals," IEEE Microw. Wireless Compon. Lett., vol. 25 , no. 2, pp. 139-141, Feb. 2015.

[8] M. Helaoui, S. Boumaiza, and F. M. Ghannouchi, "On the outphasing power amplifier nonlinearity analysis and correction using digital predistortion technique," in Proc. IEEE Radio Wireless Symp., Jan. 2008, pp. 751-754.

[9] A. Birafane and A. B. Kouki, "On the linearity and efficiency of outphasing microwave amplifiers," IEEE Trans. Microw. Theory Techn., vol. 52, no. 7, pp. 1702-1708, Jul. 2004.

[10] J. Fritzin, A. Alvandpour, P. N. Landin, and C. Fager, "Linearity, intermodulation distortion and ACLR in outphasing amplifiers," in IEEE MTT-S Int. Microw. Symp. Dig., Jun. 2013, pp. 1-4.

[11] W. Gerhard and R. Knoechel, "Nonlinear behaviour and digital phase predistortion of outphasing power amplifiers," in Proc. German Microw. Conf. (GeMIC), Hamburg, Germany, Mar. 2008, pp. 1-4.

[12] J. Fritzin, Y. Jung, P. N. Landin, P. Handel, M. Enqvist, and A. Alvandpour, "Phase predistortion of a class-D outphasing RF amplifier in $90 \mathrm{~nm}$ CMOS," IEEE Trans. Circuits Syst. II, Exp. Briefs, vol. 58, no. 10, pp. 642-646, Oct. 2011.

[13] P. N. Landin, J. Fritzin, W. Van Moer, M. Isaksson, and A. Alvandpour, "Modeling and digital predistortion of class-D outphasing RF power amplifiers," IEEE Trans. Microw. Theory Techn., vol. 60, no. 6, pp. 1907-1915, Jun. 2012
[14] P. N. Landin, T. Eriksson, J. Fritzin, and A. Alvandpour, "Behavioral modeling of outphasing amplifiers considering memory effects," in IEEE MTT-S Int. Microw. Symp. Dig., Jun. 2013, pp. 1-4.

[15] A. H. Wright, M. D. Vose, K. A. De Jong, and L. M. Schmitt, "Foundations of genetic algorithms," in Proc. 8th Int. Conf. Found. Genet. Algorithms, Aizuwakamatsu, Japan, 2005, pp. 187-200.

[16] V. J. Mathews and G. L. Sicuranza, Polynomial Signal Processing, 1st ed. Hoboken, NJ, USA: Wiley, 2000, pp. 1-4.

[17] P. Z. Marmarelis and K.-I. Naka, "Identification of multi-input biological systems," IEEE Trans. Biomed. Eng., vol. BME-21, no. 2, pp. 88-101, Mar. 1974.

[18] E. B. Solovyeva, "Piecewise-piecewise-polynomial and cascade models of predistorter for linearization of power amplifier," Radioelectron. Commun. Syst., vol. 55, no. 8, pp. 375-380, Aug. 2012.

[19] A. Zhu, P. J. Draxler, C. Hsia, T. J. Brazil, D. F. Kimball, and P. M. Asbeck, "Digital predistortion for envelope-tracking power amplifiers using decomposed piecewise Volterra series," IEEE Trans. Microw. Theory Techn., vol. 56, no. 10, pp. 2237-2247, Oct. 2008.

[20] S. Afsardoost, T. Eriksson, and C. Fager, "Digital predistortion using a vector-switched model," IEEE Trans. Microw. Theory Techn., vol. 60 , no. 4, pp. 1166-1174, Apr. 2012.

[21] F. Bação, V. Lobo, and M. Painho, "Self-organizing maps as substitutes for k-means clustering," in Proc. 5th Int. Conf. Comput. Sci., Atlanta, GA, USA, 2005, pp. 476-483.

[22] T. Kohonen, "The self-organizing map," Proc. IEEE, vol. 78, no. 9, pp. $1464-1480$, Sep. 1990.

[23] F. Maiorana, "Performance improvements of a Kohonen self organizing classification algorithm on sparse data sets," in Proc. 10th WSEAS Int. Conf. Math. Methods, Comput. Techn. Intell. Syst., Corfu, Greece, 2008, pp. $347-352$.

[24] V. Kekatos, D. Angelosante, and G. B. Giannakis, "Sparsity-aware estimation of nonlinear Volterra kernels," in Proc. 3rd IEEE Int. Workshop Comput. Adv. Multi-Sensor Adapt. Process. (CAMSAP), Aruba, Dutch Antilles, Dec. 2009, pp. 129-132.

[25] K. Finnerty, J. Dooley, and R. Farrell, "Utilizing sparse-aware Volterra for power amplifier behavioral modeling," in Proc. 17th Res. Colloq. Commun. Radio Sci. 21st Century, Dublin, Ireland, 2014, pp. 1-4.

[26] R. Tibshirani, "Regression shrinkage and selection via the lasso," J. Roy. Statist. Soc. B (Methodol.), vol. 58, no. 1, pp. 267-288, 1996.

[27] G. H. Golub, M. Heath, and G. Wahba, "Generalized cross-validation as a method for choosing a good ridge parameter," Technometrics, vol. 21, no. 2, pp. 215-223, May 1979.

[28] D. C. Lay, Linear Algebra and Its Applications. Reading, MA, USA: Addison-Wesley, 2004, pp. 364-365.

[29] M. Isaksson, D. Wisell, and D. Ronnow, "A comparative analysis of behavioral models for RF power amplifiers," IEEE Trans. Microw. Theory Techn., vol. 54, no. 1, pp. 348-359, Jan. 2006.

Keith Finnerty (GS'15), photograph and biography not available at the time of publication.

John Dooley, photograph and biography not available at the time of publication.

Robin Wesson, photograph and biography not available at the time of publication.

Mark P. van der Heijden (S'00-M'04), photograph and biography not available at the time of publication.

Mustafa Acar (M'09), photograph and biography not available at the time of publication.

Ronan Farrell (S'92-A'98-M'00), photograph and biography not available at the time of publication. 